\title{
Metalexikographische Methoden in der Computerlexikographie
}

\footnotetext{
$1 \quad$ Einleitung

2 Forschungsbereiche der maschinellen Lexikographie

3 Maschinelle Lexikographie und Metalexikographie

4 Maschinelle Analyse von Wörterbuchtexten in der Computerlexikographie

5 META: Methode zur $A$ rtikelstrukturanalyse für das Wörterbuchparsing

5.1 Funktional-positionale Segmentation und Artikelstrukturgrammatiken

5.2 Analyse von Wörterbuchtexten mit META
}

5.1.1 Schritt 1: Entwicklung der Mikrostrukturgrammatik

5.2.2 Schritt 2: Entwicklung der Artikelstrukturgrammatik

5.2.3 Schritt 3: Entwicklung der parserspezifischen Grammatik

5.2.4 Schritt 4: Testen und Verbessern der parserspezifischen Grammatik

5.3 Unterschiede zwischen metalexikographischer und computerlinguistischer Analyse von Artikelstrukturen

Literatur

\section{Einleitung}

Bislang hat die mit dem Aufbau von Lexika für Sprachverarbeitungssysteme befaßte Computerlexikographie metalexikographische Forschungsergebnisse nur wenig zur Kenntnis genommen. Die theoriegeleitete Erforschung der Bauteile und Strukturen von Wörterbuchtexten ist jedoch eine wichtige Voraussetzung dafür, daß Wörterbücher in Wörterbuchdatenbanken überführt werden können, die als Datengrundlage sowohl beim Aufbau von Lexika für die maschinelle Sprachverarbeitung als auch beim Aufbau von Hypertext-Wörterbüchern für menschliche Benutzer herangezogen werden

Der vorliegende Artikel versteht sich als Plädoyer für die Relevanz metalexikographischer Forschungsergebnisse für die computerlexikographische Praxis. Zunächst werden die Forschungsbereiche Computerlexikographie und computerunterstützte Lexikographie gegeneinander abgegrenzt; dann wird deren Verhältnis zur lexikographischen Praxis einerseits und zur Metalexikographie andererseits skizziert. Der Hauptteil der Arbeit zeigt am Beispiel des sog. Wörterbuchparsings, wie metalexikographische Methoden und Forschungsergebnisse in der computerlinguistischen Praxis umgesetzt werden können.

\section{Forschungsbereiche der maschinellen Lexikographie}

Die Bedeutung, die dem Aufbau hochwertiger Lexika in der maschinellen Sprachverarbeitung zukommt, und die vielfältigen Veränderungen, die der Vormarsch der Computertechnik für die lexikographische Praxis mit sich bringt, läßt es sinnvoll erscheinen, den bislang sehr weit gefaßten Bereich der maschinellen Lexikographie in die Teilbereiche computerunterstützte Lexikographie und Computerlexikographie zu unterteilen: ${ }^{1}$

1 Die Zusammenhänge sind in Abbildung 1 verdeutlicht. 


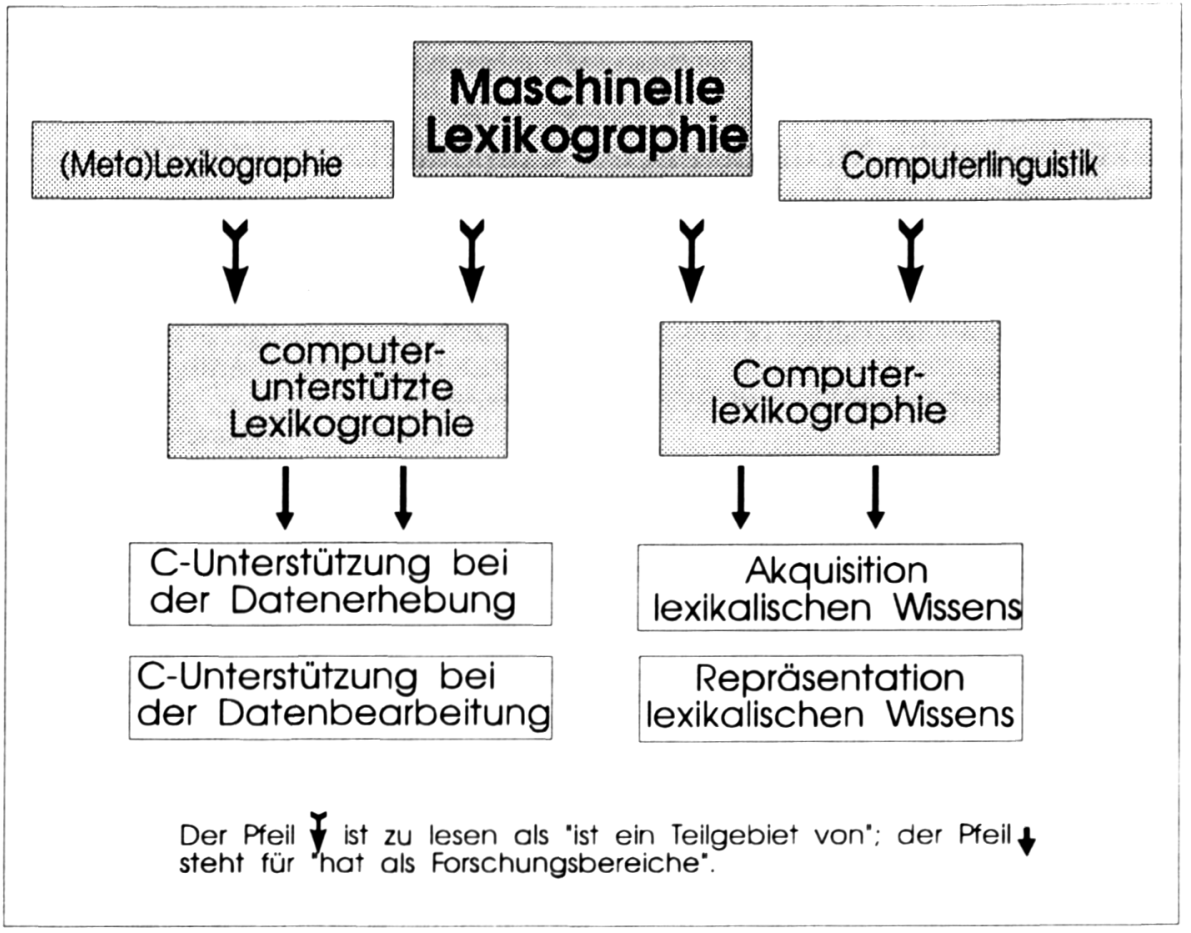

Abb. 1: Maschinelle Lexikographie

Die computerunterstützte Lexikographie beschäftigt sich mit den Möglichkeiten, den lexikographischen Arbeitsprozeß in all seinen Phasen durch den Computer zu unterstützen. Endprodukt dieses Prozesses sind Wörterbücher für menschliche Benutzer, die als Buch oder mit „Neuen Medien“ vertrieben werden. Motivation für den Einsatz von Computern bei der Datenerhebung und Datenbearbeitung ist die Beschleunigung und Erleichterung der Arbeitsprozesse, die im Idealfall zu qualitativ besseren und aktuelleren Wörterbüchern führt.

Die Computerlexikographie befaßt sich mit der Spezifikation lexikalischen Wissens für Systeme der maschinellen Sprachverarbeitung, z.B. Systeme zur Generierung gesprochener Sprache, Systeme zur Analyse geschriebener Texte oder Systeme zur maschinellen Übersetzung.

Die Entwicklung von Lexikonkomponenten für maschinelle Sprachverarbeitungssysteme erfolgte lange Zeit - teilweise heute noch - nicht in eigenständigen Projekten, sondern war eng an die Entwicklung des Gesamtsystems gekoppelt. Die lexikalischen Angaben in solchen Lexika sind determiniert vom Typ des Sprachverarbeitungssystems, dem zugrundegelegten sprachtheoretischen Modell und der vom System benutzten Repräsentationssprache für linguistisches Wissen. Durch ihre Gebundenheit an ein bestimmtes Sprachverarbeitungssystem können solche Computerlexika nicht oder nur unter großem Aufwand für andere Anwendungen nutzbar gemacht werden; vielmehr müssen dieselben lexikalischen Angaben für verschiedene Systeme immer wieder neu kodiert werden. Dies führt in letzter Konsequenz zu dem als „lexikalischer Flaschenhals“ bezeichneten Problem, daß die Erhöhung der Performanz von Sprachverarbeitungssystemen umfangreiche und qualitativ hochwertige Computerlexika erfor- 
dert, deren Aufbau jedoch aus Zeit- und Kostengründen oft nicht angemessen betrieben werden kann. Dieses Dilemma hat ab Mitte der 80er Jahre die Computerlexikographie zu einem wichtigen, eigenständigen Teilgebiet der Computerlinguistik werden lassen, wobei die beiden folgenden Problembereiche im Rahmen nationaler und internationaler Projekte erforscht werden:

Repräsentation lexikalischen Wissens: Wie kann lexikalisches Wissen so repräsentiert werden, daß es für unterschiedliche Anwendungen der maschinellen Sprachverarbeitung genutzt werden kann? Erforscht werden einerseits die Möglichkeiten und Grenzen einer polytheoretischen, multifunktionalen Beschreibung lexikalischen Wissens, ${ }^{2}$ andererseits werden konkrete Standardisierungsvorschläge vorgelegt, ${ }^{3}$ die den Austausch lexikalischer Daten zwischen verschiedenen Systemen zumindest erleichtern sollen.

Akquisition lexikalischen Wissens: Wie können vorhandene Quellen linguistischen Wissens für den Aufbau qualitativ hochwertiger Computerlexika nutzbar gemacht werden? Hierbei sucht man einerseits nach Verfahren, maschinenlesbaren Textkorpora mit Hilfe statistischer Verfahren lexikalisches Wissen abzugewinnen, ${ }^{4}$ andererseits wird geprüft, inwieweit bereits klassifizierte, computerlesbare Sprachdaten, also Computerlexika und maschinenlesbare Wörterbücher, für den Aufbau neuer Computerlexika „wiederverwendet" werden können.

Durch die Forschungen im Bereich der Akquisition lexikalischen Wissens ergeben sich Überschneidungen zwischen den Interessen der Computerlexikographie und denen der computerunterstützten Lexikographie:

Die computerlexikographische Forschung ist daran interessiert, maschinenlesbare Formen von Wörterbüchern für den Aufbau von Lexika für die maschinelle Sprachverarbeitung zu nutzen. Obwohl die in Wörterbüchern für menschliche Benutzer kodierten lexikographischen Angaben für die meisten Anwendungen der maschinellen Sprachverarbeitung nicht ausreichend und nicht explizit genug sind, liefern sie doch einen guten Grundstock an Informationen, der dann entsprechend intellektuell nachbearbeitet werden kann. ${ }^{6}$ Die teilweise mangelhafte Qualität vieler manuell erstellter Computerlexika reflektierend, wird außerdem versucht, beim Aufbau von Computerlexika stärker als bislang auf die langjährige Erfahrung der praktischen Lexikographie zurückzugreifen.

Die computerunterstützte praktische Lexikographie hat ihrerseits ein zunehmendes Interesse, vorhandene Wörterbuchdaten in computerlesbare Form zu überführen. Die in der Computerlexikographie erstellten Wörterbuchdatenbanken sind nicht nur Datengrundlage fur die o.g. computerlexikographischen Experimente im Bereich Akquisition, sondern auch wertvolle Vorläufer für ,intelligente“ Formen von elektronischen Wörterbüchern. Weiterhin können die Wörterbuchdatenbanken auch als Komponenten eines computerunterstützten Lexikographiesystems fungieren, die ein komfortables Editieren und Bearbeiten von Wörterbucheinträgen unterstützen. ${ }^{7}$

Auch bei der computerunterstützten Datenerhebung und Datenbeschreibung ergeben sich Berührungspunkte: Werkzeuge, die große Mengen von Textkorpora mit statistischen Verfahren auswerten und so die Beschreibung lexikalischer Daten empirisch abstützen können, eröffnen vor allem bei der Erfassung der Kombinatorik lexikalischer Einheiten (Mehrwortlexeme,

\footnotetext{
Z.B. StOrRer 1990, Caroli et al. 1990, Briscoe 1991, SchÜtZ \& Caroli 1991.

Z.B. SPERBERG-MCQUEEN \& BURNARD 1994; CALZOLARI 1995.

Z.B. CHURCH et al. 1991; SINCLAIR 1991

Z.B. Bläser et al. 1992; HeId \& McNAUGHT 1991; HeYN 1992; STORRER \& SCHWALl 1995.

Vgl. die Untersuchungen in BOGURAEV \& BRISCOE 1989 und die Evaluierung in IDE \& VERONIS 1995.

Vgl. BLÄSER \& WERMKE 1990.
} 
Kollokation) neuartige Recherchemöglichkeiten, die für die computergestützte Lexikographie und Computerlexikographie gleichermaßen interessant sind ${ }^{8}$

\section{Maschinelle Lexikographie und Metalexikographie}

Die computerunterstützte Lexikographie ist bereits seit WIEGAND 1983 potentieller Gegenstand metalexikographischer Theoriebildung. Zunächst tentativ der Teiltheorie der lexikographischen Spracherforschung zugeschlagen, sind mittlerweile nahezu alle der in WIEGAND 1983 aufgeführten Forschungsgebiete (vgl. Abb. 2) vom Einzug des Computers betroffen.

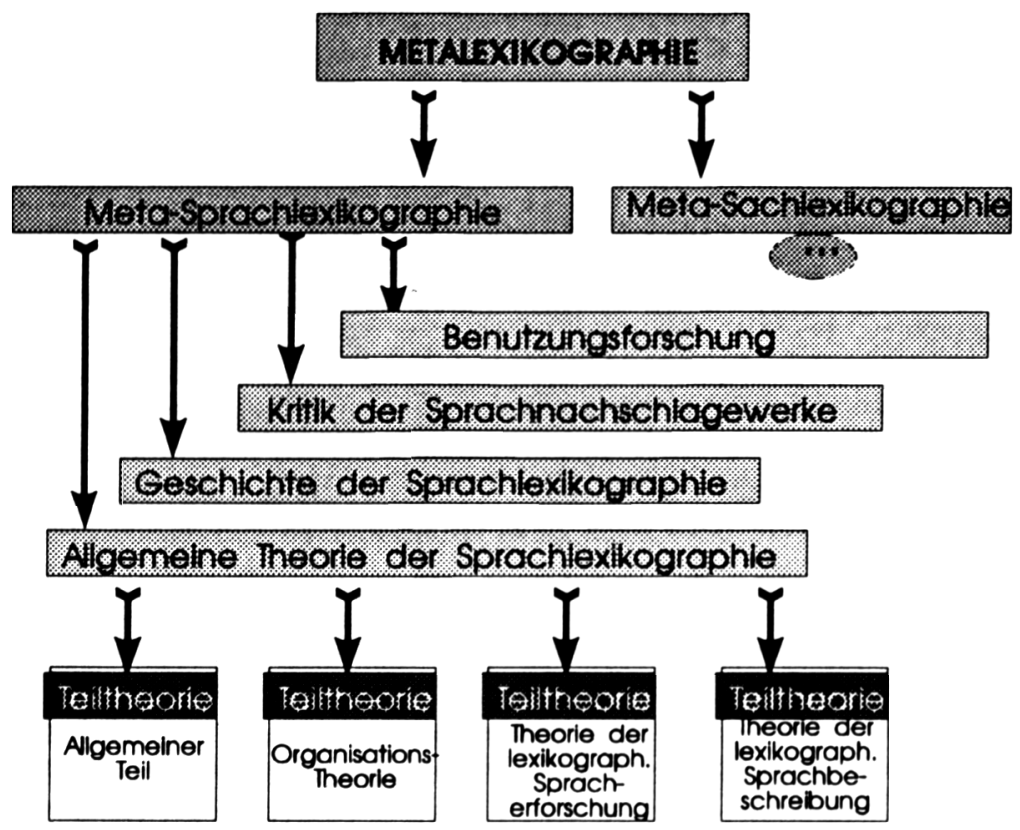

Abb. 2: Metalexikographische Forschungsbereiche

Die Publikation von Wörterbuchdaten auf „Neuen Medien“ eröffnet nicht nur neue Märkte, sondern auch andere Möglichkeiten des Zugriffs auf die Wörterbuchdaten und damit auch neue Formen der Wörterbuchbenutzung. ${ }^{9}$ Richtlinien für bedienerfreundliche und vielfältig verwendbare elektronische Wörterbücher zu erarbeiten, stellt die Benutzungsforschung vor eine neue Herausforderung.

Die Wörterbuchkritik verfügt über völlig neuartige Möglichkeiten der quantitativen und qualitativen Auswertung von Wörterbuchdaten, sofern diese in computerlesbarer und entspre-

8 Vgl. Сhurch \& Hanks 1990; Smadja 1993; für das Deutsche: BreidT 1993.

9 Vgl. STORRER 1995 und LeHr \& KAMMERER in diesem Band. 
Treffen, das: 1. Zusammenkunft. Begegnung: regelmäßige, seltene T.; ein T. der Abiturienten; ein T. der Außenminister; ein T. verabreden, veranstalten; an einem T. teilnehmen; zu einem T. kommen. 2. (militär. veraltet) Gefecht: frische Truppen ins T. führen. 3. (Sport) Wettkampf: ein faires, spannendes T.; das T. endete unentschieden; sie konnte das $T$. für sich entscheiden. * (geh.:) etwas ins Treffen führen (etwas als Argument vorbringen).

Abkomen, eine Absprache t. $\partial 2 \hat{u}$ (etwas vereinba\}-\}

ren). Dvr10ix

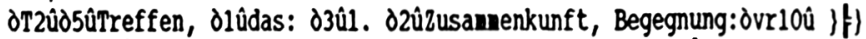

olûregeln hige, seltene T.; ein T. der Abiturienten; \}F\}

ein T. der A h enninister; ein T. verabreden, ver $\}-\}$

anstalten; an eines T. teilnehnen; $2 \mathrm{u}$ einer T. \} H \}

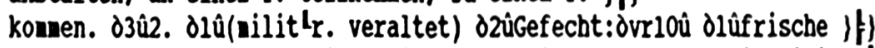

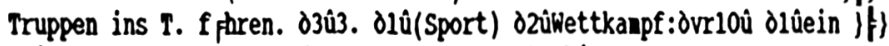

faires, spannendes T.; das T. endete unentschie\}-\}

den; sie konnte das $\mathrm{I}$. $\mathrm{f}_{\mathrm{f}} \mathrm{r}$ sich entscheiden. \} F $\}$

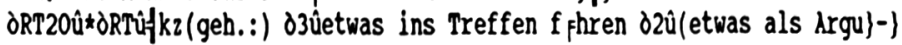

nent vorbringen). ovr10üe

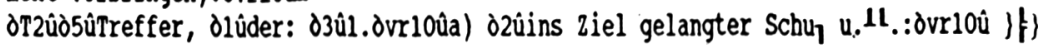

Abb. 3: Wörterbuchartikel und Satzbandtext zum Lemma Treffen im DUDEN-2

chend strukturierter Form vorliegen. ${ }^{10}$ Außerdem wird sie sich künftig auch mit nur elektronisch publizierten Wörterbüchern kritisch auseinandersetzen müssen.

Der Veränderungen, die sich durch die zunehmende Computerisierung des lexikographischen Arbeitsprozesses auf die Organisationstheorie, die Theorie der lexikographischen Spracherforschung und die Theorie der lexikographischen Sprachbeschreibung ergeben, wurden in Abschnitt 2 bereits angesprochen.

Während die computerunterstützte Lexikographie also von jeher ein metalexikographischer Untersuchungsgegenstand ist, haben sich Metalexikographie und Computerlexikographie bislang kaum zur Kenntnis genommen. Dabei sind metalexikographische Forschungsergebnisse extrem hilfreich, wenn es darum geht, Experimente zur (halb)automatischen Extraktion lexikalischen Wissens aus Wörterbuchdatenbanken zu konzipieren und - vor allem - die Grenzen solcher Extraktionsexperimente vorherzusagen und zu erklären

Im folgenden wird gezeigt, daß auch für die Konversion von Wörterbuchdaten in eine Wörterbuchdatenbank, die - wie oben gezeigt - für computergestützte Lexikographie und Computerlexikographie gleichermaßen von entscheidender Bedeutung ist, metalexikographi-

10 Vgl. HEYN 1992; HeYN et al. 1992. 
Intry $\|$ accepted.

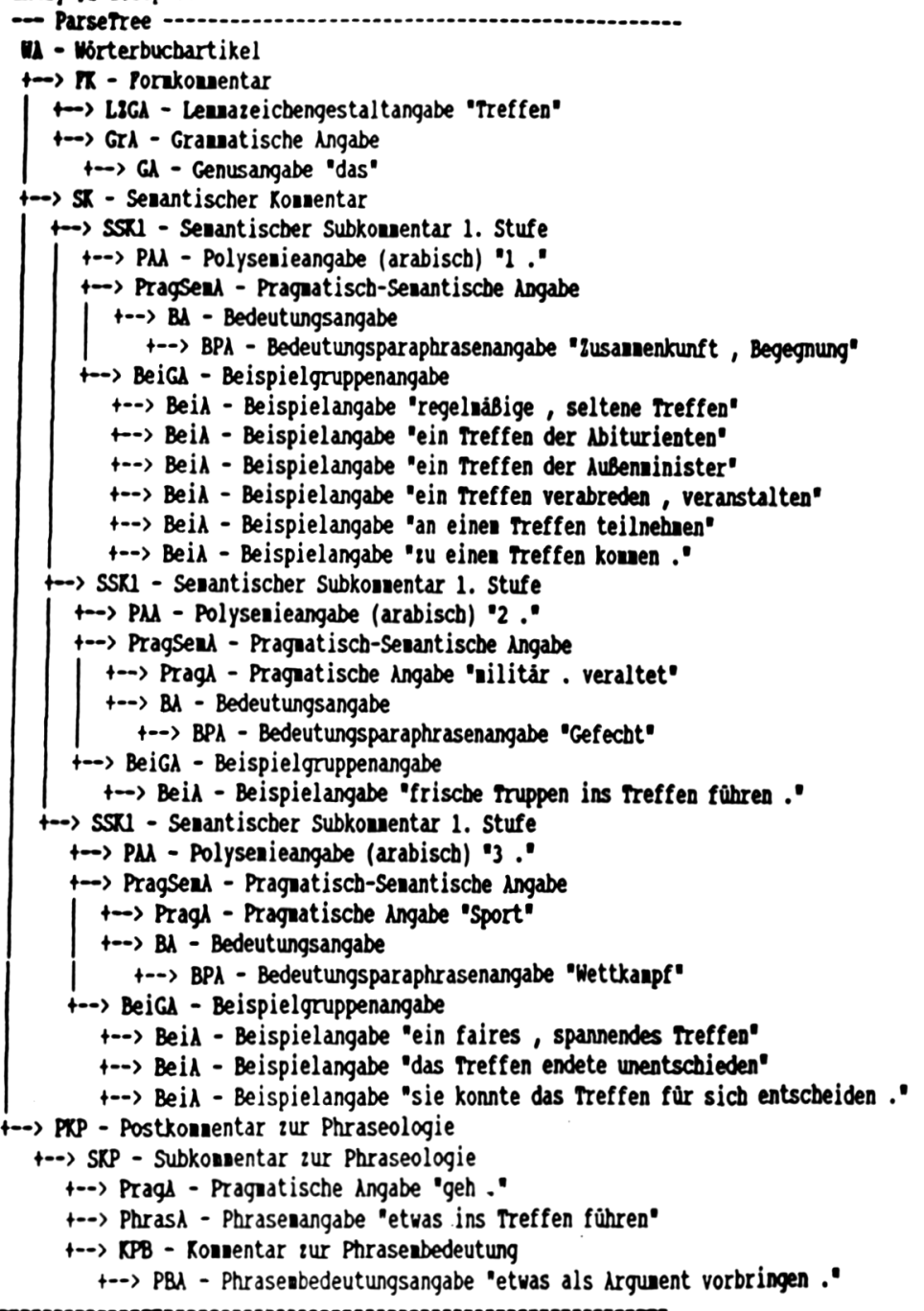

Abb. 4: Parsebaum zum Lemma Treffen im DUDEN-2

sche Forschungsergebnisse und Methoden von großem Nutzen sind, wenn sie entsprechend an die computerlexikographische Praxis adaptiert werden.

\section{Maschinelle Analyse von Wörterbuchtexten in der Computerlexikographie}

$\mathrm{Zu}$ vielen neueren Wörterbüchern gibt es sog. Satzbanddateien, die bei der Drucklegung des Wörterbuchs mittels einer Lichtsatzmaschine entstehen. Abb. 3 zeigt den Wörterbucheintrag zum Lemma Treffen des DUDEN-2; oben in der Form, in der er im Wörterbuch erscheint, un- 
ten in der Form, in der er auf dem Satzband repräsentiert ist. Man sieht, daß die Repräsentation des Wörterbuchtextes in der Satzbanddatei stark vom Layout des Wörterbuchs und den idiosynkratischen Steuerkodes der Lichtsatzmaschine geprägt ist. In dieser Form kann der Wörterbuchtext weder in computerlinguistischen Extraktionsexperimenten verwendet werden noch ist er für die computerunterstützte Lexikographie - etwa für eine Neubearbeitung des Wörterbuchs - geeignet. Um die Satzbandrepräsentationen von Wörterbüchern maschinell in ein zur Wiederverwendung geeignetes Format zu überführen, wurden in der Computerlexikographie sog. Wörterbuchparser entwickelt. Ein Wörterbuchparser ist ein Programmsystem, das einen auf Satzband gespeicherten Wörterbuchtext in seine zentralen Gliederungseinheiten, die Wörterbuchartikel, zerlegt und diesen dann, relativ zu einer vorgegeben Artikelstrukturgrammatik, eine Strukturbeschreibung zuweist. Abb. 3 zeigt die Strukturbeschreibung zum Lemma Treffen, wie sie von dem Wörterbuchparser LEXPARSE (HAUSER \& STORRER 1994) nach erfolgreicher maschineller Strukturanalyse des Satzbandtextes ausgegeben wird. Es wird deutlich, $\mathrm{da} ß$ in einer solchen Repräsentation alle lexikographischen Angaben einer bestimmten Angabenklasse zugeordnet sind, so daß entsprechende Computerprogramme gezielt Struktur- und Wertebereichsanalysen durchführen können. Aus diesem Grund werden Wörterbücher, deren Artikel in dieser Weise repräsentiert sind, auch als Wörterbuchdatenbanken bezeichnet.

Bislang standen bei der Diskussion des Wörterbuchparsings computerlinguistische Fragen, z.B. nach Systemfunktionalität, effizienten Parsingstrategien und geeigneten Grammatikformalismen, im Vordergrund. ${ }^{11}$ Die Frage, wie bei der Entwicklung von Grammatiken für das Wörterbuchparsing methodisch vorzugehen sei, wurde nur am Rande behandelt. Für die praktische Arbeit des Wörterbuchparsings ist aber die Analyse der Wörterbuchartikel und die Entwicklung einer solchen Grammatik eine zentrale Aufgabe, die zudem nicht den Entwicklern des Parsingsystems überlassen werden kann, sondern die Zusammenarbeit zwischen Systementwicklern und metalexikographisch geschulten (Computer)lexikographen erfordert. Um eine sinnvolle Arbeitsteilung zwischen diesen Gruppen zu ermöglichen und die Diskussion über die Analyseergebnisse zu erleichtern, wird neben einer einheitlichen Terminologie für die zu analysierenden Gegenstände eine Methode benötigt, die die zu bewältigende Aufgabe in Teilschritte zerlegt. Eine solche Methode möchte ich im folgenden zur Diskussion stellen.

\section{META: Methode zur Artikelstrukturanalyse für das Wörterbuchparsing}

META wurde in dem an der Universität Tübingen durchgeführten ELWIS-Projekt ${ }^{12}$ zur korpusunterstützten Entwicklung lexikalischer Wissensbasen entwickelt und erprobt. Der ursprüngliche Zweck von META war es, die im Projekt beschäftigten Hilfskräfte bei der Entwicklung von Artikelstrukturgrammatiken für die vorhandenen Wörterbuchdaten ${ }^{13}$ anzuleiten und das methodische Vorgehen in computerlinguistischen Seminaren an Studierende vermitteln zu können. Die Methode ist jedoch generell für alle Projekte geeignet, die mit dem Parsen von Wörterbüchern befaßt sind.

11 Vgl. Neff \& Boguraev 1990; Bläsi \& Koch 1992; Hauser 1993; Hauser \& StORrer 1994

12 Vgl. STORRER et al. 1994.

13 Es handelt sich um Teile der Satzbänder des Duden-2 und des Duden-10; unser Dank geht an Dr. M. WERMKE und die Duden-Redaktion, die uns diese Daten für Forschungszwecke zur Verfugung gestellt haben. 
Das vierschrittige Vorgehen in META orientiert sich zunächst am gedruckten Wörterbuchtext und geht erst im dritten Schritt über zu einer ergänzenden Analyse des Satzbandes. Gegenüber der direkten Analyse des Satzbandtextes hat dies folgende Vorteile:

Wie aus der in Abb. 3 gezeigten Gegenüberstellung von Wörterbuchtext und Satzbandtext unschwer ersichtlich wird, ist der Aufbau der Wörterbuchartikel am Wörterbuchtext wesentlich besser erkennbar. Häufig läßt sich die Funktion von Segmenten des Satzbandtextes überhaupt nur durch den Vergleich mit dem Wörterbuchtext zuordnen. Die in Schritt 3 zu vollziehende Unterscheidung zwischen strukturrelevanten und strukturirrelevanten Segmenten des Satzbandtextes setzt bereits eine genaue Analyse der gedruckten Form des Wörterbuchtextes voraus.

Die Methode ermöglicht prinzipiell eine Arbeitsteilung zwischen (Meta)lexikographen und Systementwicklern: Da die Schritte 1 und 2 auf dem gedruckten Wörterbuch basieren und noch unabhängig von einem bestimmten Wörterbuchparser sind, können sie von den Lexikographen selbst durchgefürt werden, sofern diese in der Lage sind, Teil-Ganzes und Abfolgebeziehungen zwischen lexikographischen Textsegmenten mit Hilfe kontextfreier Ersetzungsregeln zu formulieren. Auf dieser Basis können sich die Systementwickler in den Schritten 3 und 4 dann auf die Lösung spezieller Parsingprobleme konzentrieren, wobei die in Schritt 4 erfolgende Fehleranalyse wiederum Rückkopplung mit den Lexikographen erfordert.

Die Analyse des gedruckten Wörterbuchtextes kann auf der Grundlage der Methode der funktional-positionalen Segmentation erfolgen, die von H.E. WIEGAND im Rahmen seiner Theorie lexikographischer Texte zur Analyse von Wörterbuchstrukturen vorgeschlagen wurde. ${ }^{14}$ Die furr das Parsing wichtige Trennung in Angaben, typographische und nichttypographische Strukturanzeiger (s.u.), wird durch die Abfolge der methodischen Schritte reflektiert.

Nach einer kurzen Einführung in die metalexikographischen Grundbegriffe, die für das Verständnis der META-Methode unabdingbar sind, ${ }^{15}$ werden in den folgenden Abschnitten die methodischen Schritte im einzelnen besprochen und die Unterschiede zwischen computerlexikographischer und metalexikographischer Analyse von Wörterbuchtexten herausgestellt.

\subsection{Funktional-positionale Segmentation und Artikelstrukturgrammatiken}

Nach der Methode der funktional-positionalen Segmentation werden Wörterbuchartikel so lange in funktionale Textsegmente zerlegt, bis die entstehenden Segmente unter funktionalem Gesichtspunkt nicht mehr weiter segmentierbar sind. Dabei lassen sich zwei Typen von Textsegmenten unterscheiden: Lexikographische Angaben und nicht-typographische Strukturanzeiger, mit denen Angaben und Angabegruppen voneinander abgegrenzt werden. Aus den lexikographischen Angaben lassen sich Informationen zum Wörterbuchgegenstand erschließen; diese können - in Abhängigkeit von dem mit der Angabe verfolgten Zweck - disjunkten Klassen von Angaben zugeordnet werden. Nichttypographische Strukturanzeiger und typographische Strukturanzeiger, d.h., die Schriftarten und Schriftypen, in denen Angaben bestimmter Klassen erscheinen, fungieren als Orientierungshilfen, durch die ein rasches und gezieltes Auffinden der gesuchten Information unterstützt wird.

14 Wir orientieren uns an der Darstellung der Methode in WIEGAND 1991a, Abschnitt 4.1.

15 Die nachfolgenden Erläuterungen zu metalexikographischen Termini wie Mikrostruktur, Strukturanzeiger und lexikographische Angabe haben die Funktion, die Grundideen von META auch für diejenigen verstehbar zu machen, die nicht mit der Theorie lexikographischer Texte vertraut sind. Die Erläuterungen sind deshalb oft stark vereinfachend; für die exakten Definitionen verweisen wir auf die entsprechende Spezialliteratur. 
Zwischen den funktionalen Textsegmenten eines Wörterbuchartikels bestehen verschiedene strukturprägende Relationen, von denen zwei für die in META durchzuführenden Analysen relevant sind:

Eine Teil-Ganzes-Relation, die auf der Menge der funktionalen Textsegmente definiert ist und damit die partitive Artikelstruktur festlegt.

Eine Vorgänger-Nachfolger-Relation, die auf der Menge der elementaren, nicht weiter segmentierbaren funktionalen Textsegmente definiert ist und damit die präzedentive Artikelstruktur festlegt.

Beide Relationen gemeinsam determinieren die konkrete hierarchische Artikelstruktur eines Wörterbuchs. ${ }^{16}$ Während die konkrete Artikelstruktur alle funktionalen Textsegmente, also auch die nichttypographischen Strukturanzeiger berücksichtigt, wird die konkrete hierarchische Mikrostruktur eines Wörterbuchartikels lediglich durch Teil-Ganzes- und VorgängerNachfolger-Beziehungen zwischen lexikographischen Angaben konstituiert.

Die Methode der funktional-positionalen Segmentation trägt dieser Unterscheidung durch verschiedene Varianten Rechnung: ${ }^{17}$

- Bei der nicht-exhaustiven Variante der funktional-positionalen Segmentation werden nur lexikographische Angaben berücksichtigt, d.h., die Segmentation resultiert in der konkreten hierarchischen Mikrostruktur des segmentierten Wörterbuchartikels.

- Bei der exhaustiven Variante der funktional-positionalen Segmentation werden neben den lexikographischen Angaben auch die nicht-typographischen Strukturanzeiger berücksichtigt, d.h., die Segmentation resultiert in der konkreten hierarchischen Artikelstruktur des segmentierten Wörterbuchartikels.

- Die exhaustive Variante der funktional-positionalen Segmentation mit Isolierung der typographischen Strukturanzeiger berücksichtigt zusätzlich die Schriftarten und Schriftschnitte, in denen Angaben oder nichttypographische Strukturanzeiger erscheinen.

Für die Entwicklung von Grammatiken für das Wörterbuchparsing muß nun von den konkreten Mikro- und Artikelstrukturen abstrahiert und zu generelleren Strukturbeschreibungen übergegangen werden. Durch Zuordnung der lexikographischen Angaben zu Angabeklassen kann von konkreten Mikrostrukturen zu abstrakten Mikrostrukturen bzw. von konkreten Artikelstrukturen zu abstrakten Artikelstrukturen übergegangen werden.

Ein weiterer Abstraktionsschritt führt von den abstrakten Mikrostrukturen verschiedener Wörterbuchartikel zur Spezifikation einer Mikrostrukturgrammatik, die idealiter alle wohlgeformten abstrakten hierarchischen Mikrostrukturen der im Wörterbuch verzeichneten Wörterbuchartikel erzeugt bzw. lizensiert. Eine solche Mikrostrukturgrammatik für ein Wörterbuch $w_{b}$ kann als eine kontextfreie Grammatik MSG $\left(w_{b}\right)\langle K N A, K E A, R, W A\rangle$ spezifiziert werden; ${ }^{18}$ hierbei ist:

KNA: Menge der Klassen nicht-elementarer lexikographischer Angaben

KEA: Menge der Klassen elementarer lexikographischer Angaben

$\mathrm{R}=$ Menge kontextfreier Ersetzungsregeln

WA $($ Wörterbuchartikel $)=$ das Startsymbol $\in$ KNA .

Der Unterschied zwischen abstrakter Mikrostruktur und abstrakter Artikelstruktur besteht vereinfacht gesprochen - darin, daß erstere über Klassen von lexikalischen Angaben, letztere über Klassen von Angaben und nichttypographischen Strukturanzeigern definiert wird. Eine

16 Für eine detaillierte, formale Beschreibung von Strukturen in Artikeltexten verweisen wir auf WIEGAND $1989 \mathrm{~b}$ und WIEGAND 1989c.

17 Vgl. WIEGAND 1991a:22.

$18 \mathrm{Zu}$ den formalen Eigenschaften kontextfreier Grammatiken und ihrer maschinellen Verarbeitung vgl. PARTEE et al. 1990, Kap. 18. 


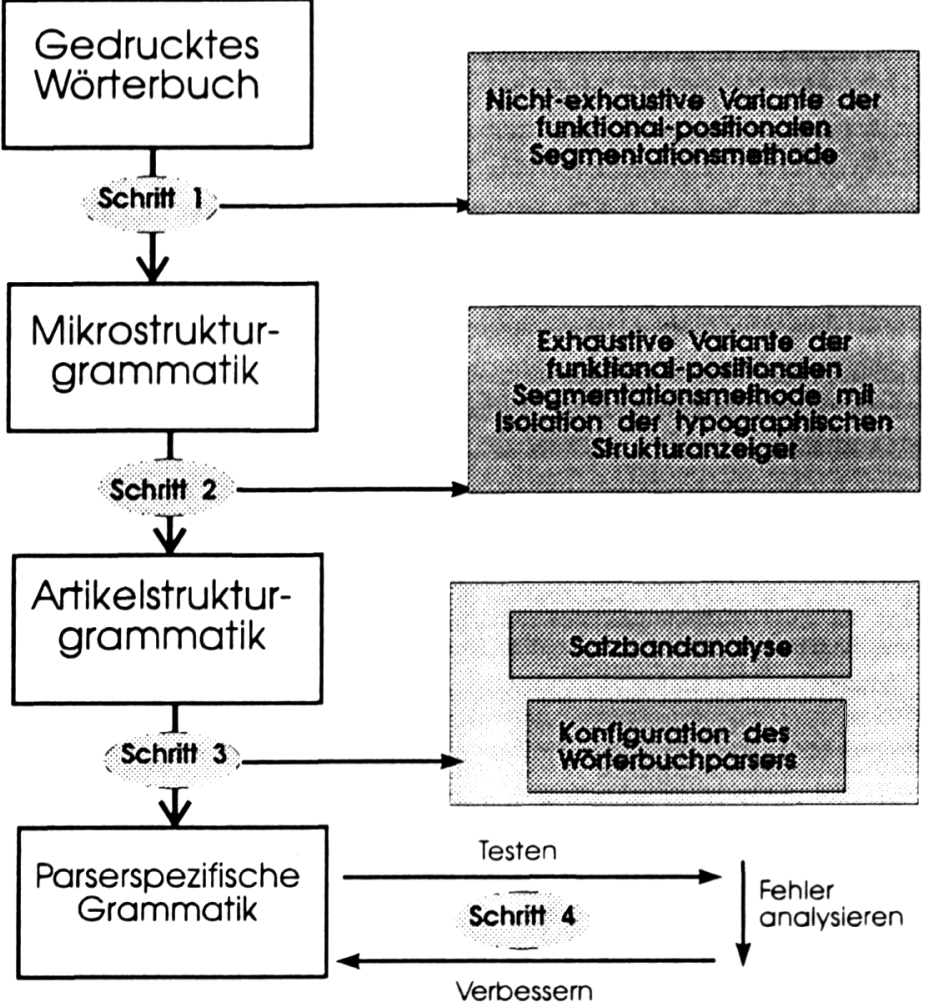

Abb. 5: META - Methode zur Artikelstrukturanalyse für das Wörterbuchparsing

Artikelstrukturgrammatik für das Wörterbuch $w_{\mathrm{i}}$, die idealiter alle wohlgeformten abstrakten hierarchischen Artikelstrukturen der in $w b_{i}$ verzeichneten Wörterbuchartikel erzeugt bzw. lizensiert, läßt sich also spezifizieren als eine kontextfreie Grammatik $\operatorname{ASG}\left(w_{b}\right)\langle K N T, K T T, R$, WA); hierbei ist

KNT: Menge der Klassen nicht-elementarer lexikographischer Textsegmente

KTT: Menge der Klassen terminaler lexikographischer Textsegmente

$\mathbf{R}=$ Menge kontextfreier Ersetzungsregeln

WA (Wörterbuchartikel) $=$ das Startsymbol $\in$ KNT.

Prinzipiell können Mikrostrukturgrammatiken und Artikelstrukturgrammatiken auch in anderer Weise formal spezifiert werden; kontextfreie Grammatiken bieten sich furr unsere Zwecke jedoch deshalb an, weil der Umgang mit kontextfreien Ersetzungsregeln relativ einfach erlernbar ist und zum (computer)linguistischen Grundwissen gehört; die entstehenden Grammatikbeschreibungen sind übersichtlicher und damit besser diskutierbar als die parserspezifischen Notationen. 


\subsection{Analyse von Wörterbuchtexten mit META}

Die methodischen Schritte in META sind in Abb. 5 veranschaulicht.

- Schritt 1 führt von der Analyse des gedruckten Wörterbuchtextes unter Anwendung der nicht-exhaustiven Variante der funktional-positionalen Segmentation zum Gerüst einer Mikrostrukturgrammatik.

- In Schritt 2 wird dieses Gerüst durch Anwendung der exhaustiven Variante der funktional-positionalen Segmentation zum Gerüst einer Artikelstrukturgrammatik erweitert.

- Schritt 3 geht über vom gedruckten Wörterbuchtext zur Analyse des Satzbandes und überführt die ASG in eine parserspezifische Grammatik.

- In Schritt 4 wird das Satzband geparst; die entstehenden Strukturbeschreibungen sind im Hinblick auf ihre Adäquatheit zu überprüfen und die parserspezifische Grammatik oder die fehlerhaften Wörterbuchartikel sind entsprechend zu verändern. Diese Testläufe werden so lange wiederholt, bis allen Wörterbuchartikeln eine korrekte Strukturbeschreibung zugeordnet werden kann.

Als Vorarbeit sollte zunächst der Metatext zum Artikelaufbau des Wörterbuchs genau gelesen und die in ihm erläuterten Klassen lexikographischer Angaben aufgelistet werden. Da die Termini zur Bezeichnung der Angabeklassen in verschiedenen Wörterbüchern differieren, ist es sinnvoll, eine einheitliche Terminologie festzulegen und die wörterbuchspezifischen Termini in diese zu übersetzen. Die in den Arbeiten zur metalexikographischen Theorie lexikographischer Texte eingeführten Termini ${ }^{19}$ sind hierfür besonders gut geeignet, da sie - im Gegensatz zum meist unvollständigen Metatext der Wörterbücher - Termini für alle Textsegmente bereitstellen, die für die Analyse relevant werden, also auch für komplexe Textsegmente wie Formkommentar, semantischer Kommentar und verschiedene Postkommentare. Ergebnis der Metatext-Lektüre sind eine Liste von Bezeichnungen für Klassen von Angaben, die laut Metatext in den Wörterbüchern verzeichnet sind und die bei den Analysen in Schritt 1 zugrundegelegt werden, sowie eine (meist unvollständige) Liste der Angaben zu Form und Funktion der im Wörterbuch verwendeten typographischen und nicht-typographischen Strukturanzeiger, die für Schritt 2 und 3 der META-Methode benötigt werden. Im folgenden werden die Schritte im einzelnen beschrieben und einige der dabei auftretenden Probleme diskutiert

\subsubsection{Schritt 1: Entwicklung der Mikrostrukturgrammatik}

Im ersten Schritt werden konkrete hierarchische Mikrostrukturen von Wörterbuchartikeln zu verschiedenen Lemmazeichentypen untersucht. Über die Zuordnung der Angaben zu Angabeklassen und die Analyse der Teil-Ganzes- und Vorgänger-Nachfolger-Beziehungen kann ein Gerüst für eine Mikrostrukturgrammatik des Wörterbuchs erstellt werden. Schritt 1 besteht wiederum aus drei Teilschritten:

Im ersten Teilschritt werden einfach strukturierte Artikel für verschiedene Lemmazeichentypen ausgewählt und in elementare, d.h. nicht weiter segmentierbare lexikographische Angaben zerlegt. Jede dieser elementaren Angaben wird einer funktional bestimmten Klasse lexikographischer Angaben zugeordnet. Ergebnis dieses Teilschrittes ist ein zunächst unvollständiges Inventar der Klassen elementarer Angaben KEA, die terminalen Symbole der Mikrostrukturgrammatik

Im zweiten Teilschritt werden die elementaren Angaben der analysierten Wörterbuchartikel unter funktionalen Gesichtspunkten zu komplexen Angaben kombiniert. Diese können wiederum als Bausteine einer höheren Komplexitätsstufe analysiert werden, bis hin zur höchsten Komplexitätsstufe, dem Wörterbuchartikel selbst, dem Startsymbol der Mikrostrukturgrammatik. Jede dieser komplexen Angaben wird nun einer Klasse funktional bestimmten Angaben zu-

19 Vgl. WIEgAND 1989a; WIEGAND 1989b; WIEgAND 1991b; WIEGAND 1994. 
geordnet. Die Art und Weise, wie sich Klassen von Angaben zu Klassen von Angaben einer höheren Komplexitätsstufe zusammensetzen lassen, wird in kontextfreien Ersetzungsregeln festgehalten. Ergebnisse dieses Teilschrittes sind also ein zunächst unvollständiges Inventar der Klassen nicht-elementarer Angaben KNA, die als nicht-terminale Kategorien in die Mikrostrukturgrammatik eingehen, und eine ebenfalls unvollständige Menge kontextfreier Ersetzungsregeln.

Das Vorgehen bei der Analyse der einzelnen Wörterbuchartikel entspricht dabei der nichtexhaustiven Variante der Methode der funktional-positionalen Segmentation. Während bei dieser allerdings nicht-elementare und elementare Angaben durch eine Top-down-Analyse in einem Schritt gewonnen werden, werden in der hier vorgeschlagenen Vorgehensweise im ersten Teilschritt zunächst die elementaren Angaben, d.h. die Terminale der Strukturanalyse bestimmt. Erst im zweiten Teilschritt werden dann im Bottom-up-Verfahren die komplexen Einheiten ermittelt. Diese Abwandlung erwies sich bei der Vermittlung der Methode an Studierende der Computerlinguistik als erfolgreicher als das Top-down-Vorgehen, bei dem bereits ein Vorwissen über funktionale Einheiten von Wörterbuchartikeln vorausgesetzt wird. (Meta)lexikographisch geschulte Personen hingegen, die einen Wörterbuchartikel bereits mit geübtem Strukturblick betrachten, werden mit dem ursprünglich vorgeschlagenen Top-down-Verfahren vermutlich schneller ans Ziel gelangen.

Im dritten Teilschritt werden die Inventare der terminalen und der nicht-terminalen Kategorien und die bislang ermittelten Ersetzungsregeln der im Aufbau befindlichen Mikrostrukturgrammatik durch Analyse weiterer Wörterbuchartikel vervollständigt. Ziel ist es, möglichst viele der möglichen Variations- und Ausbaumöglichkeiten für komplexe funktionale Textsegmente (z.B. Postkommentar zur Phraseologie, Beispielgruppenangabe, pragmatisch-semantische Angabe) zu entdecken und das Grundgerüst der Mikrostrukturgrammatik entsprechend auszubauen. Bei diesem Teilschritt ist es sinnvoll, Gruppen von Wörterbuchartikeln zum selben Lemmazeichentyp zusammenzustellen, wobei sich in dieser Phase meist bereits ein guter Blick für strukturell interessante Wörterbuchartikel herausgebildet hat. Ist das Grundgerüst der Mikrostrukturgrammatik hinreichend umfangreich, d.h., werden komplex strukturierte Wörterbuchartikel zu allen Lemmazeichentypen von ihr lizensiert, kann zum zweiten Schritt der META-Methode übergegangen werden.

\subsubsection{Schritt 2: Entwicklung der Artikelstrukturgrammatik}

Ziel des zweiten Schrittee ist es, das Inventar der in den Wörterbuchartikeln verwendeten nicht-typographischen und typographischen Strukturanzeiger und deren Funktion zu ermitteln und damit das Gerüst der Mikrostrukturgrammatik zum Gerüst einer Artikelstrukturgrammatik zu erweitern. Dies geschieht, indem die im ersten Schritt gewonnenen Mikrostrukturen einfacher Wörterbuchartikel in zwei Teilschritten re-analysiert werden:

Der erste Teilschritt orientiert sich an der exhaustiven Variante der Methode der funktional-positionalen Segmentation. Die Re-Analyse konzentriert sich dabei auf die Ermittlung des Inventars der nicht-typographischen Strukturanzeiger, durch die die lexikographischen Angaben umschlossen oder voneinander abgetrennt werden. Diese sind dann an entsprechender Stelle in die Ersetzungsregeln der Mikrostrukturgrammatik zu integrieren. Nicht-typographische Strukturanzeiger gehören zur Menge der terminalen Einheiten KTT, da sie zwar Teil einer Klasse komplexer Angaben sein können, aber nicht untereinander zu komplexen Strukturanzeigern kombinierbar sind. Die Mikrostrukturgrammatik wird durch diesen Teilschritt zu einer Artikelstrukturgrammatik im oben definierten Sinne ausgebaut 
Der zweite Teilschritt konzentriert sich auf die typographischen Strukturanzeiger, d.h. die Schriftarten und Schriftschnitte, in denen die lexikographischen Textsegmente, also die Angaben und die nicht-typographischen Strukturanzeiger, erscheinen. Alle Textsegmente, die Träger eines spezifischen typographischen Strukturanzeigers sind, werden mit einem typographischen Index versehen. Da typographische Strukturanzeiger ihre strukturanzeigende Funktion nur mit Hilfe der lexikographischen Textsegmente ausüben können, an die sie gebunden sind, ${ }^{20}$ gehören sie nicht zu den terminalen und nicht-terminalen Kategorien der Artikelstrukturgrammatik, können aber über Indizierung der Klassen der elementaren und nicht-elementaren Textsegmente KET bzw. KTT in der Artikelstrukturgrammatik berücksichtigt werden.

Indem alle in Schritt 1 analysierten Wörterbuchartikel auf diese Weise re-analysiert werden, entsteht ein stabiles Gerüst einer Artikelstrukturgrammatik, in dem sowohl typographische als auch nicht-typographische Strukturanzeiger berücksichtigt sind.

\subsubsection{Schritt 3: Entwicklung der parserspezifischen Grammatik}

In diesem Schritt wird vom gedruckten Wörterbuchtext zur Analyse des Satzbandtextes übergegangen. Auf dem Hintergrund dieser Analyseergebnisse und der Artikelstrukturgrammatik kann dann der Wörterbuchparser konfiguriert werden. Auch hierbei lassen sich zwei Teilschritte unterscheiden:

Im ersten Teilschritt muß durch den Vergleich von Wörterbuch- und Satzbandtext die Funktion der satzbandspezifischen Steuerzeichen identifiziert werden. Die Sequenzen im Satzbandtext, die für die maschinelle Erkennung der Artikelstruktur irrelevant sind (z.B. Steuerzeichen für Seitenumbruch, Kopfzeilen etc.), müssen von den Sequenzen mit strukturanzeigender Funktion unterschieden werden. Neben den Steuercodes, die Anfang und Ende eines Wörterbuchartikels kennzeichnen, sind für das Wörterbuchparsing die sog. Fontcodes, d.h. Satzbandsequenzen, die die Umschaltung in eine bestimmte Schriftart oder einen bestimmten Schriftyp induzieren, besonders relevant.

Im zweiten Teilschritt wird die Artikelstrukturgrammatik in den Grammatikformalismus übersetzt, der vom jeweiligen Parsingsystem verarbeitet werden kann. Hierbei ist die Art und Weise zu berücksichtigen, in der die Wörterbuchartikel im Satzbandtext repräsentiert werden, d.h., unabhängig von dem eingesetzten Wörterbuchparser müssen die strukturirrelevanten Sequenzen ignoriert und die im Satzband verwendeten Steuercodes korrekt interpretiert werden können. Dies erfordert gerade im Bereich der typographischen Strukturanzeiger, die in der Artikelstrukturgrammatik deklarativ als Indizes der lexikographischen Textsegmente kodiert sind, einiges Umdenken: Fontcodes, das Pendant der typographischen Strukturanzeiger im Satzbandtext, sind nicht deklarativ, sondern prozedural zu interpretieren. Sie fungieren als Schalter, durch die in eine bestimmte Schriftart oder einen Schriftschnitt umgeschaltet wird. So bewirkt beispielsweise der Fontcode „òlû“ im Satzbandtext des DUDEN-2 eine Umschaltung auf den Standard-Schriftschnitt, der Fontcode „ò2û“ eine Umschaltung auf den kursiven Schriftschnitt. Durch welche Ereignisse eine solche Umschaltung außer Kraft gesetzt wird, ist abhängig vom jeweiligen Fontcode; dies muß bei der Konfiguration der Wörterbuchgrammatik berücksichtigt werden. ${ }^{21}$ Weiterhin stellt sich bei der Übersetzung in die parserspezifische Grammatik auch oft heraus, daß die Tiefe, die bei der maschinellen Analyse der Artikelstruktur erreicht werden kann, begrenzt ist. Dies liegt daran, daß die maschinelle Identifikation der lexikographischen Textsegmente nur anhand der Strukturanzeiger erfolgen kann, die auf dem Satzband identifi-

20 Vgl. Wiegand 1991a:26f.

21 Wie mit diesem Problem beim Wörterbuchparsing umgegangen werden kann, ist in HaUSER \& STORRER 1994 an Beispielen erörtert. 
ziert werden können, und nicht - wie bei der intellektuellen Segmentation - durch das Verständnis des Wörterbuchgegenstands gestützt ist. Im Vergleich zur intellektuell erstellten Artikelstrukturgrammatik müssen deshalb meist Abstriche an die Analysetiefe in Kauf genommen werden.

Nach der Konfiguration des Wörterbuchparsers kann ein erster Durchlauf der Satzbanddateien erfolgen und zu Schritt 4 übergegangen werden.

\subsubsection{Schritt 4: Testen und Verbessern der parserspezifischen Grammatik}

Die letzte Phase besteht darin, die parserspezifische Grammatik kontinuierlich zu erweitern und zu verbessern. Dies geschieht durch die Analyse der Wörterbuchartikel, denen keine Strukturbeschreibung zugeschrieben werden konnte oder deren Strukturbeschreibung nicht mit (meta)lexikographischen Intuitionen übereinstimmt. Korrekturen und Testläufe werden so lange wiederholt, bis alle auf dem Satzband gespeicherten wohlgeformten Wörterbucheinträge korrekt geparst sind. Die Einschränkung „alle wohlgeformten Wörterbuchartikel“ trägt der Tatsache Rechnung, daß auf den Satzbändern meist auch Wörterbuchartikel zu finden sind, in denen Strukturanzeiger fehlen oder in inkonsistenter Weise verwendet werden. In diesen Fällen muß natürlich nicht die parserspezifische Grammatik verändert, sondern das Satzband korrigiert werden. Wie lange es dauert, bis eine 100prozentige Erkennungsrate erreicht ist, hängt von der Komplexität des Wörterbuchs und der Explizitheit der verwendeten Strukturanzeiger ab. Die Dauer der letzten Phase kann jedoch erheblich verkürzt werden, wenn die parserspezifische Grammatik, die dem ersten Testlauf zugrundeliegt, schon relativ reichhaltig ist, d.h., wenn die in Schritt 1 und 2 erarbeitete Artikelstrukturgrammatik schon alle wesentlichen Strukturvarianten berücksichtigt.

\subsection{Unterschiede zwischen metalexikographischer und computerlinguistischer Analyse von Artikelstrukturen}

Die Artikelstrukturanalysen in META dienen dem Zweck, möglichst rasch eine Grammatik zu erstellen, mit der ein auf Satzband repräsentiertes Wörterbuch korrekt, effizient und möglichst vollständig geparst werden kann. Dies unterscheidet sie von metalexikographischen Analysen mit theoretisch ausgerichteter Zielsetzung, beispielsweise der Typologisierung existierender und möglicher Strukturen und Strukturierungsprinzipien und deren Bewertung relativ zu Wörterbuchtypen und/oder Wörterbuchbenutzungssituationen. Wegen der unterschiedlichen Ziele, die mit der Artikelstrukturanalyse in Metalexikographie und Computerlexikographie verfolgt werden, müssen die metalexikographischen Methoden und Termini an die computerlexikographische Praxis adaptiert werden:

Wie bereits erwähnt, müssen bei der maschinellen Strukturanalyse Abstriche an die Analysetiefe gemacht werden, da die lexikographischen Textsegmente nur anhand der Strukturanzeiger identifiziert werden können und nicht, wie bei der intellektuellen Analyse des gedruckten Wörterbuchtextes, anhand des Verständnisses von Inhalt und Funktion der lexikographischen Angaben. Die parserspezifische Grammatiken für Wörterbücher mit wenigen oder inkonsistent verwendeten Strukturanzeigern können dann nur Teile der intellektuell erstellten Artikelstrukturgrammatiken wiedergeben, so daß solche Wörterbücher nur mit Mühe und großem Nachbereitungsaufwand in eine Wörterbuchdatenbank überführbar sind.

Die in META erstellten Grammatiken müssen nicht nur korrekt sein, sondern auch effizient verarbeitet werden können, d.h, die Anzahl der Ersetzungsregeln und die Inventare der terminalen und nicht-terminalen Kategorien sollten möglichst klein gehalten werden. Falls die Feingliederung eines Textsegments (z.B. die Segmentation einer Deklinationsangabe in eine 
Singularbildungsangabe und eine Pluralbildungsangabe) eine Reihe komplizierter Regeln erforderlich macht, kann es u.U. sinnvoll sein, auf diese zunächst zu verzichten und die Deklinationsangabe als terminale Einheit der Strukturdarstellung unanalysiert zu belassen.

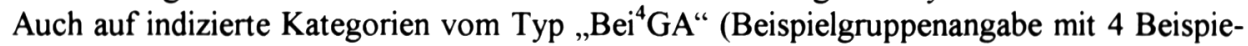
len) sollte verzichtet werden, da sie den Regelapparat durch gleichartige Ersetzungsregeln aufblähen, die nur in der Anzahl der Tochterknoten (in diesem Fall der Anzahl der Beispielangaben) variieren. Die in der Indizierung enthaltene Information der Anzahl der Tochterknoten läßt sich ohnehin - bei Bedarf - in einem Nachbearbeitungsschritt aus der Strukturbeschreibung berechnen.

Weiterhin sollte man bei der maschinellen Strukturanalyse auf Kategorien verzichten, deren maschinelle Identifikation eine Binnenanalyse des Angabetextes erfordern würde: So läßt sich die Phrasemangabe PhrasA im DUDEN-2 relativ gut anhand der typographischen und nichttypographischen Strukturanzeiger erkennen. Für eine feinere Kategorisierung vom Typ PhrAs/2v/A (= Phrasemangabe, aus der 2 Phrasemvarianten erschließbar sind) würden wesentlich kompliziertere Regeln benötigt. Aus diesem Grund ist es zweckmäßiger, derartige Binnenanlysen in einem dem eigentlichen Parsing nachgeschalteten Schritt durchzuführen. ${ }^{22}$

Aus Effizienzgründen ist es auch nicht erstrebenswert, die Ersetzungsregeln der Mikro- und Artikelstrukturgrammatiken in Abhängigkeit vom Typ des Lemmazeichens zu formulieren, wenngleich der Zusammenhang von Artikelstruktur und Lemmazeichentyp im methodischen Verfahren berücksichtigt wird. Vielmehr müssen die gemeinsamen Aufbauprinzipien für verschiedene Lemmazeichentypen in möglichst generellen Regeln erfaßt werden, die dann je nach Lemmazeichentyp unterschiedlich expandiert werden.

Insgesamt profitiert die META-Methode von metalexikographischen Forschungsergebnissen in methodischer und terminologischer Hinsicht: In der Theorie lexikographischer Texte sind für die meisten Gegenstände und Beziehungen, die für Wörterbuchparsing relevant sind, metalexikographisch fundierte Termini eingeführ, mit der sich die Analyseergebnisse in einheitlicher Weise darstellen und diskutieren lassen. Die Methode der funktional-positionalen Segmentation in ihren verschiedenen Varianten ermöglicht ein theoriegeleitetes Vorgehen bei der Grammatikerstellung.

\section{Literatur}

BLÄSER, B.; WERMKE, M. (1990): Projekt „Elektronische Wörterbücher/Lexika“. Abschlußbericht der Definitionsphase. IWBS-Report 145, IBM Deutschland, Stuttgart.

Bläser, B.; U. Schwall; A. StoRRer (1992): A Reusable Lexical Database for Machine Translation. In: Proceedings of the Twelfth International Conference on Computational Linguistics COLING, Nantes, 510516.

BLÄSI, C.; КосH, H.-D. (1992): Maschinelle Strukturerschließung von Wörterbuchartikeln mit Standardmethoden. In: Lexicographica 7 (1991), 182-201.

BREIDT, E. (1993): Extraktion von Verb-Nomen-Verbindungen aus dem Mannheimer Korpus I. SfS-Report 0393, Universität Tübingen, Seminar für Sprachwissenschaft.

Briscoe, B. (1991): Lexical Issues in Natural Language Processing. In: KLEIN, E.; VeltmanN, F. (Hg.) (1991): Natural Language and Speech. Berlin, Heidelberg, 39-69.

Boguraev, B.; BrISCOE, T. (Hg.) (1989): Computational Lexicography for Natural Language Processing. London New York.

CALZOLARI, N. (1995): European Efforts Towards Standardizing Language Resources. In: STEFFENS 1995, 121130.

22 Zur Binnenanalyse von Phrasemangaben vgl. STORRER \& SCHWALL 1995. 
Caroli, F.; Haller, J.; SchÜtz, J.; TRUaR, M. (1990): Wiederverwendbarkeit lexikalischer Ressourcen. In: SCHAEDER, B.; RIEGER, B. (ed.) (1990): Lexikon und Lexikographie. Grundlagen - Entwicklungen - Produkte. Hildesheim et al., 62-78.

Church, K.W.; Hanks, P. (1990): Word Association Norms, Mutual Information and Lexicography. In: Computational Linguistics 16 (1990), 22-29.

Church, K.W.; Gale, W.A.; Hanks, P.; Hindle, D.M. (1991): Using Statistics in Lexical Analysis. In ZERNIK, U. (ed.): Lexical Acquisition: Exploring On-line Resources to Build a Lexicon. Hillsdale NJ., 115164.

Duden-2: Stilwörterbuch der deutschen Sprache. Die Verwendung der WöRter im Satz. 7. völlig neu bearbeitete Auflage; Hg.: G. DrosDowkI, Mannheim, Wien, Zürich 1990.

DUdEN-10: Das BedeutungswörTERBUCh. WORTBILDUNG UND WORTSChaTZ. 2. völlig neu bearb. Auflage. Hg.: W. MỨlLeR Mannheim, Wien, Zürich 1985.

Hauser, R. (1993): Das Parsen von Wörterbüchern - Problematik und Lösungsansätze. Magisterarbeit. Universität Tübingen, Seminar für Sprachwissenschaft.

Hauser, R., Storrer, A. (1994): Dictionary Entry Parsing Using the LeXParse System. In: Lexicographica 9 (1993), 174-219.

Hausmann, F.J.; Reichmann, O.; Wiegand, H.E.; Zgusta, L. (Hg.) (1989): Wörterbücher. Ein internationales Handbuch zur Lexikographie. 1. Teilbd. Berlin, New York.

HeID, U.; McNaUght, J. (1991): EUROTRA-7 Study: Feasibility and Project Definition Study on the Reusability of Lexical and Terminological Resources in Computerized Applications. Final report, CEC-DG XIII, Luxemburg.

HEYN, M. (1992): Zur Wiederverwendung maschinenlesbarer Wörterbücher - Eine computergestützte metalexikographische Studie am Beispiel der elektronischen Edition des „Oxford Advanced Learner's Dictionary of Current English“. Tübingen 1992.

HEYN, M.; CHRIST, O.; HEID, U. (1992): Computergestützte Metalexikographie. Erfahrungen bei der Ermittlung der Wiederverwendbarkeit eines Wörterbuchs für die maschinelle Sprachverarbeitung. In: LDV-Forum 9/1 (1992), 23-33.

IDE, N.; VERONIS, J. (1995): Knowledge Extraction from Machine-Readable Dictionaries: An Evaluation. In STEFFENS 1995, 19-34.

NefF, M.; BoguraEv, B. (1990): From Machine Readable Dictionaries to Lexical Databases. Report IBM T.J. Watson Research Center, New York.

Partee, B.H.; Meulen, A.; Wall, R. (1990): Mathematical Methods in Linguistics. Dordrecht, Boston, London.

SchưtZ, J.; CAROLI, F. (1991): An Architecture for Reusable Lexical Resources in a Multi-theoretical Environment. DOC-8 of the EUROTRA-7 study. Technical report, CEC-DG XIII, Luxemburg.

SINCLAIR, J. (1991): Corpus, Concordance, Collocation. Oxford et al.

SMADJA, F.A. (1993): Retrieving Collocations from Text: Xtract. In: Computational Linguistics, 19/1 (1993). 143-177.

SPERBERG-MCQueEN, C.M.; BurnaRD, L. (Hg.) (1994): Guidelines for Electronic Text Encoding and Interchange. TEI P3. 2Bd. Text Encoding Initiative Chicago Oxford.

STEFFENS, P. (Hg.) (1995): Machine Translation and the Lexicon. Proceedings of the Third International EAMT Workshop in Heidelberg, Germany, April 1993, Berlin Heidelberg.

STORRER, A. (1990): Überlegungen zur Repräsentation der Verbsyntax in einer multifunktional-polytheoretischen lexikalischen Datenbank. In: SchAEDER, B.; RIEGER, B. (ed.) (1990): Lexikon und Lexikographie. Grundlagen - Entwicklungen - Produkte. Hildesheim et al., 120-133.

STORRER, A. (1995): Wörterbücher zum Anklicken. Ein kleiner Rundgang durch die PC-Bibliothek. In: Sprachreport, 2/95, Mannheim.

STORRER, A.; FELDWEG, H.; HINRICHS, E.W. (1994): Korpusunterstützte Entwicklung lexikalischer Wissensbasen. In: Sprache und Datenverarbeitung 17 (1993), Bonn, 59-72.

StORRer, A.; Schwall, U. (1995): Description and Acquisition of Multiword Lexemes. In: STEFfens 1995. 35-50.

WIEGAND, H.E. (1983) Ansätze zu einer allgemeinen Theorie der Lexikographie. In: SCHILDT, J.; VIEHWEgER, D. (1983) (Hg.): Die Lexikographie von heute und das Wörterbuch von morgen. Analysen - Probleme Vorschläge. Linguistische Studeien, Reihe A, Arbeitsberichte 109, Berlin, 92-127. 
WIEGAND, H.E. (1989a): Aspekte der Makrostruktur im allgemeinen einsprachigen Wörterbuch: alphabetische Anordnungsformen und ihre Probleme. In: HAUSMANN et al. (1989), 371-409.

WiEgand, H.E. (1989b): Der Begriff der Mikrostruktur: Geschichte, Probleme, Perspektiven. In: Hausmann et al. (1989), 409-462.

WIEGAND, H.E. (1989c): Formen von Mikrostrukturen im allgemeinen einsprachigen Wörterbuch. In: HAUSMANN et al. (1989), 462-501.

WiEgAND, H.E. (1991a): Printed Dictionaries and their Parts as Text. An Overview of More Recent Research as an Introduction. In: Lexicographica 6 (1990), 1-124.

WIEGAND, H.E. (1991b): Über die Strukturen der Artikeltexte im Frühneuhochdeutschen Wörterbuch. Zugleich ein Versuch zur Weiterentwicklung einer Theorie lexikographischer Texte. In: GoEBEL, U, REICHMANN, O. (Hg.): Historical Lexicography of the German Language. Vol. 2, Lewiston, Queenston, Lampeter, 341-673.

WIEGAND, H.E. (1994): Elements of a Theory towards a so-called Lexicographic Definition. In: Lexicographica 8 (1992), 175-289.

Angelika Storrer, Institut für deutsche Sprache, Postfach 101621, 68016 Mannheim 